\title{
Article \\ Bounds for the Differences between Arithmetic and Geometric Means and Their Applications to Inequalities
}

\author{
Shigeru Furuichi ${ }^{1}\left(\mathbb{D}\right.$ and Nicuşor Minculete ${ }^{2, *}$ \\ 1 Department of Information Science, College of Humanities and Sciences, Nihon University, Setagaya-ku, \\ Tokyo 156-8550, Japan; furuichi.shigeru@nihon-u.ac.jp \\ 2 Department of Mathematics and Computer Science, Transilvania University of Braşov, \\ 500091 Braşov, Romania \\ * Correspondence: minculete.nicusor@unitbv.ro
}

check for updates

Citation: Furuichi, S.; Minculete, N. Bounds for the Differences between Arithmetic and Geometric Means and Their Applications to Inequalities. Symmetry 2021, 13, 2398. https:// doi.org/10.3390/sym13122398

Academic Editors: Theodore E. Simos and Sergei D. Odintsov

Received: 7 November 2021 Accepted: 10 December 2021 Published: 12 December 2021

Publisher's Note: MDPI stays neutral with regard to jurisdictional claims in published maps and institutional affiliations.

Copyright: (C) 2021 by the authors. Licensee MDPI, Basel, Switzerland. This article is an open access article distributed under the terms and conditions of the Creative Commons Attribution (CC BY) license (https:// creativecommons.org/licenses/by/ $4.0 /)$.

\begin{abstract}
Refining and reversing weighted arithmetic-geometric mean inequalities have been studied in many papers. In this paper, we provide some bounds for the differences between the weighted arithmetic and geometric means, using known inequalities. We improve the results given by FuruichiGhaemi-Gharakhanlu and Sababheh-Choi. We also give some bounds on entropies, applying the results in a different approach. We explore certain convex or concave functions, which are symmetric functions on the axis $t=1 / 2$.
\end{abstract}

Keywords: Shannon entropy; Tsallis entropy; Fermi-Dirac entropy; Bose-Einstein entropy; arithmetic mean; geometric mean; Young's inequality

MSC: 26D20; 94A15

\section{Introduction}

We denote a set of all probability distributions by

$$
\Delta_{n}:=\left\{\mathbf{p}=\left\{p_{1}, p_{2}, \cdots, p_{n}\right\} \mid p_{j}>0,(j=1,2, \cdots, n), \sum_{j=1}^{n} p_{j}=1\right\} .
$$

In this manuscript, for mathematical simplicity we remove the case $p_{j}=0$ for $j=$ $1,2, \cdots, n$. For any $\mathbf{p} \in \Delta_{n}$, Shannon entropy $H(\mathbf{p})$, Rényi entropy $R_{q}(\mathbf{p})$ and Tsallis entropy $H_{q}(\mathbf{p})$ are defined as [1-3]

$$
H(\mathbf{p}):=-\sum_{j=1}^{n} p_{j} \log p_{j}, \quad R_{q}(\mathbf{p}):=\frac{1}{1-q} \log \left(\sum_{j=1}^{n} p_{j}^{q}\right), \quad H_{q}(\mathbf{p}):=-\sum_{j=1}^{n} p_{j}^{q} \ln _{q} p_{j} .
$$

where $\ln _{q}(x):=\frac{x^{1-q}-1}{1-q}$ is $q$-logarithmic function defined for $x>0$ and $q>0$ with $q \neq 1$. It is known that $\lim _{q \rightarrow 1} R_{q}(\mathbf{p})=\lim _{q \rightarrow 1} H_{q}(\mathbf{p})=H(\mathbf{p})$. An interesting differential relation of the Rényi entropy [4] is

$$
\frac{d R_{q}(\mathbf{p})}{d q}=-\frac{1}{(1-q)^{2}} \sum_{j=1}^{n} v_{j} \log \frac{v_{j}}{p_{j}}
$$

which is proportional to Kullback-Leibler divergence, where $v_{j}=p_{j}^{q} / \sum_{j=1}^{n} p_{j}^{q}$.

In [5], the Fermi-Dirac-Tsallis entropy was introduced by

$$
I_{q}^{F D}(\mathbf{p}):=\sum_{j=1}^{n} p_{j} \ln _{q} \frac{1}{p_{j}}+\sum_{j=1}^{n}\left(1-p_{j}\right) \ln _{q} \frac{1}{1-p_{j}}
$$


for $\mathbf{p} \in \Delta_{n}$ and the Bose-Einstein-Tsallis entropy was given in [6] as

$$
I_{q}^{B E}(\mathbf{p}):=\sum_{j=1}^{n} p_{j} \ln _{q} \frac{1}{p_{j}}-\sum_{j=1}^{n}\left(1+p_{j}\right) \ln _{q} \frac{1}{1+p_{j}} .
$$

In the limit of $q \rightarrow 1$, we have

$$
\lim _{q \rightarrow 1} I_{q}^{F D}(\mathbf{p})=I_{1}^{F D}(\mathbf{p}):=-\sum_{j=1}^{n} p_{j} \log p_{j}-\sum_{j=1}^{n}\left(1-p_{j}\right) \log \left(1-p_{j}\right)
$$

and

$$
\lim _{q \rightarrow 1} I_{q}^{B E}(\mathbf{p})=I_{1}^{B E}(\mathbf{p}):=-\sum_{j=1}^{n} p_{j} \log p_{j}+\sum_{j=1}^{n}\left(1+p_{j}\right) \log \left(1+p_{j}\right),
$$

where $I_{1}^{F D}(\mathbf{p})$ and $I_{1}^{B E}(\mathbf{p})$ are the Fermi-Dirac entropy and the Bose-Einstein entropy, respectively. See [6] and references therein for their details.

In [7], we used the expression that describes the difference between the arithmetic mean and the weighted geometric mean:

$$
d_{p}(a, b):=p a+(1-p) b-a^{p} b^{1-p}, \quad(a, b>0, p \in[0,1]) .
$$

It is well known that $d_{p}(a, b) \geq 0$ as Young inequality or the weighted arithmeticgeometric mean inequality.

Next, we consider $d_{p}(a, b)$ for $p \in \mathbb{R}$. We easily find that the following properties:

$$
\begin{gathered}
d_{p}(a, b) \geq 0(\text { when } p \in[0,1]) \\
d_{p}(a, a)=d_{0}(a, b)=d_{1}(a, b)=0, \quad d_{p}(a, b)=d_{1-p}(b, a)
\end{gathered}
$$

and

$$
\begin{aligned}
d_{p}\left(\frac{1}{a}, \frac{1}{b}\right) & =\frac{1}{a b} d_{p}(b, a), d_{p}(a, 1)+d_{p}(b, 1) \\
& =d_{p}(a+b, 2)+2\left\{\left(\frac{a+b}{2}\right)^{p}-\frac{a^{p}+b^{p}}{2}\right\} .
\end{aligned}
$$

In [8] Sababheh and Choi prove that if $a$ and $b$ are positive numbers with $p \notin[0,1]$, then $d_{1-p}(a, b) \leq 0$.

Some important results [9-11] on the studies used to estimate bounds on several entropies have been established, recently, via the use of mathematical inequalities. We provide some results on several entropies, applying new and improved inequalities in this paper.

\section{Bounds of $d .(\cdot, \cdot)$ and Inequalities for Entropies}

We first rewrite the Tsallis entropy, Rényi entropy, the Fermi-Dirac-Tsallis entropy, and the Bose-Einstein-Tsallis entropy by the use of the notation $d .(\cdot, \cdot)$.

Lemma 1. For $\mathbf{p} \in \Delta_{n}$ and $q \geq 0$ with $q>1$, we have

(i) $H_{q}(\mathbf{p})=n-1-\frac{1}{1-q} \sum_{j=1}^{n} d_{q}\left(p_{j}, 1\right)$,

(ii) $R_{q}(\mathbf{p})=\frac{1}{1-q} \log \left\{n(1-q)+q-\sum_{j=1}^{n} d_{q}\left(p_{j}, 1\right)\right\}$,

(iii) $I_{q}^{F D}(\mathbf{p})=n-\frac{1}{1-q} \sum_{j=1}^{n}\left\{d_{q}\left(p_{j}, 1\right)+d_{q}\left(1-p_{j}, 1\right)\right\}$, 
(iv) $I_{q}^{B E}(\mathbf{p})=n-\frac{1}{1-q} \sum_{j=1}^{n}\left\{d_{q}\left(p_{j}, 1\right)-d_{q}\left(1+p_{j}, 1\right)\right\}$.

Proof. The proof can be done by the direct calculations.

(i) Simple calculations

$$
\begin{aligned}
1+H_{q}(\mathbf{p}) & =-\sum_{j=1}^{n}\left(\frac{p_{j}-p_{j}^{q}}{1-q}-p_{j}\right)=-\sum_{j=1}^{n} \frac{q p_{j}-p_{j}^{q}}{1-q}=n-\sum_{j=1}^{n}\left(\frac{q p_{j}-p_{j}^{q}}{1-q}+1\right) \\
& =n-\frac{1}{1-q} \sum_{j=1}^{n} d_{q}\left(p_{j}, 1\right)
\end{aligned}
$$

show the statement in (i).

(ii) Since we have the relation:

$$
\exp \left((1-q) R_{q}(\mathbf{p})\right)=1+(1-q) H_{q}(\mathbf{p}),
$$

we have

$$
\exp \left((1-q) R_{q}(\mathbf{p})\right)=n(1-q)+q-\sum_{j=1}^{n} d_{q}\left(p_{j}, 1\right)
$$

which implies the statement in (ii).

(iii) We can calculate as

$$
\begin{aligned}
& \sum_{j=1}^{n}\left(1-p_{j}\right) \ln _{q} \frac{1}{1-p_{j}}=\sum_{j=1}^{n} \frac{\left(1-p_{j}\right)^{q}-\left(1-p_{j}\right)}{1-q} \\
& =\sum_{j=1}^{n}\left\{\frac{\left(1-p_{j}\right)^{q}-\left(1-p_{j}\right)}{1-q}+1-p_{j}\right\}-n+1 \\
& =\sum_{j=1}^{n}\left\{\frac{\left(1-p_{j}\right)^{q}-q\left(1-p_{j}\right)}{1-q}-1\right\}+1 \\
& =1-\frac{1}{1-q} \sum_{j=1}^{n}\left\{q\left(1-p_{j}\right)+1-q-\left(1-p_{j}\right)^{q}\right\}=1-\frac{1}{1-q} \sum_{j=1}^{n} d_{q}\left(1-p_{j}, 1\right) .
\end{aligned}
$$

Thus, we have with the result of (i),

$$
\begin{aligned}
& I_{q}^{F D}(\mathbf{p})=\sum_{j=1}^{n} p_{j} \ln _{q} \frac{1}{p_{j}}+\sum_{j=1}^{n}\left(1-p_{j}\right) \ln _{q} \frac{1}{1-p_{j}} \\
& =n-1-\frac{1}{1-q} \sum_{j=1}^{n} d_{q}\left(p_{j}, 1\right)+1-\frac{1}{1-q} \sum_{j=1}^{n} d_{q}\left(1-p_{j}, 1\right) \\
& =n-\frac{1}{1-q} \sum_{j=1}^{n}\left\{d_{q}\left(p_{j}, 1\right)+d_{q}\left(1-p_{j}, 1\right)\right\} .
\end{aligned}
$$


(iv) We can calculate as

$$
\begin{aligned}
& \sum_{j=1}^{n}\left(1+p_{j}\right) \ln _{q} \frac{1}{1+p_{j}}=\sum_{j=1}^{n} \frac{\left(1+p_{j}\right)^{q}-\left(1+p_{j}\right)}{1-q} \\
& =\sum_{j=1}^{n}\left\{\frac{\left(1+p_{j}\right)^{q}-\left(1+p_{j}\right)}{1-q}+1+p_{j}\right\}-n-1 \\
& =\sum_{j=1}^{n}\left\{\frac{\left(1+p_{j}\right)^{q}-q\left(1+p_{j}\right)}{1-q}-1\right\}-1 \\
& =-1-\frac{1}{1-q} \sum_{j=1}^{n} d_{q}\left(1+p_{j}, 1\right) .
\end{aligned}
$$

Thus, we have

$$
\begin{aligned}
& I_{q}^{B E}(\mathbf{p})=\sum_{j=1}^{n} p_{j} \ln _{q} \frac{1}{p_{j}}-\sum_{j=1}^{n}\left(1+p_{j}\right) \ln _{q} \frac{1}{1+p_{j}} \\
& =n-1-\frac{1}{1-q} \sum_{j=1}^{n} d_{q}\left(p_{j}, 1\right)+1+\frac{1}{1-q} \sum_{j=1}^{n} d_{q}\left(1+p_{j}, 1\right) \\
& =n-\frac{1}{1-q} \sum_{j=1}^{n}\left\{d_{q}\left(p_{j}, 1\right)-d_{q}\left(1+p_{j}, 1\right)\right\} .
\end{aligned}
$$

We give relations on $d .(\cdot, \cdot)$.

Lemma 2. Let $a, b>0$. If $p \in \mathbb{R}$, then the following equalities hold:

$$
d_{p}(a, b)=p(\sqrt{a}-\sqrt{b})^{2}+d_{2 p}(\sqrt{a b}, b)
$$

and

$$
d_{p}(a, b)=(1-p)(\sqrt{a}-\sqrt{b})^{2}+d_{2 p-1}(a, \sqrt{a b})
$$

Proof. We note that $a^{p} b^{1-p}=(a b)^{1-p} a^{2 p-1}=(\sqrt{a b})^{2-2 p} a^{2 p-1}=(\sqrt{a b})^{2 p} b^{1-2 p}$.

(i) Then,

$$
\begin{aligned}
& d_{p}(a, b)=p a+(1-p) b-(\sqrt{a b})^{2 p} b^{1-2 p} \\
& =p a+(1-p) b-2 p \sqrt{a b}-(1-2 p) b+2 p \sqrt{a b}+(1-2 p) b-(\sqrt{a b})^{2 p} b^{1-2 p} \\
& =p(\sqrt{a}-\sqrt{b})^{2}+d_{2 p}(\sqrt{a b}, b)
\end{aligned}
$$

(ii) We also have

$$
\begin{aligned}
& d_{p}(a, b)=p a+(1-p) b-(\sqrt{a b})^{2-2 p} a^{2 p-1} \\
& =p a+(1-p) b-2(1-p) \sqrt{a b}-(2 p-1) a \\
& \quad+2(1-p) \sqrt{a b}+(2 p-1) a-(\sqrt{a b})^{2-2 p} a^{2 p-1} \\
& =(1-p)(\sqrt{a}-\sqrt{b})^{2}+d_{2 p-1}(a, \sqrt{a b}) .
\end{aligned}
$$


In several papers [7,12-14], we find estimations of the bounds of $d_{p}(a, b)$. For this purpose, we use the following inequalities from (a) to (d).

(a) Kittaneh and Manasrah gave in [12]:

$$
r(p)(\sqrt{a}-\sqrt{b})^{2} \leq d_{p}(a, b) \leq R(p)(\sqrt{a}-\sqrt{b})^{2}
$$

where $a, b>0,0 \leq p \leq 1$ and $r(p)=\min \{p, 1-p\}, R(p)=\max \{p, 1-p\}$, whose notations are used throughout this paper without mention.

(b) Cartwright and Field proved the inequality (see, e.g., [14]):

$$
\frac{1}{2} p(1-p) \frac{(a-b)^{2}}{\max \{a, b\}} \leq d_{p}(a, b) \leq \frac{1}{2} p(1-p) \frac{(a-b)^{2}}{\min \{a, b\}}
$$

for $a, b>0$ and $0 \leq p \leq 1$.

(c) Alzer, da Fonseca, and Kovačec obtained the following inequalities (see, e.g., [13]):

$$
\frac{1}{2} p(1-p) \min \{a, b\} \log ^{2} \frac{a}{b} \leq d_{p}(a, b) \leq \frac{1}{2} p(1-p) \max \{a, b\} \log ^{2} \frac{a}{b}
$$

and

$$
\min \left\{\frac{p}{q}, \frac{1-p}{1-q}\right\} d_{q}(a, b) \leq d_{p}(a, b) \leq \max \left\{\frac{p}{q}, \frac{1-p}{1-q}\right\} d_{q}(a, b),
$$

for $a, b>0$ and $0<p, q<1$.

Taking into account (1), (2) and taking $b=1$ and changing $p$ by $q$ in the above inequalities given in (a)-(c), we obtain the following.

$\left(a_{1}\right)$

$$
r(q)(\sqrt{a}-1)^{2} \leq d_{q}(a, 1) \leq R(q)(\sqrt{a}-1)^{2}
$$

$\left(b_{1}\right)$

where $a>0$ and $0 \leq q \leq 1$.

$$
\frac{1}{2} q(1-q)(a-1)^{2} \leq d_{q}(a, 1) \leq \frac{1}{2} q(1-q) \frac{(a-1)^{2}}{a}
$$

$\left(c_{1}\right)$

for $0<a \leq 1$ and $0 \leq q \leq 1$.

$$
\frac{1}{2} q(1-q) a \log ^{2} a \leq d_{q}(a, 1) \leq \frac{1}{2} q(1-q) \log ^{2} a
$$

and

$$
\min \left\{\frac{q}{p}, \frac{1-q}{1-p}\right\} d_{p}(a, 1) \leq d_{q}(a, 1) \leq \max \left\{\frac{q}{p}, \frac{1-q}{1-p}\right\} d_{p}(a, 1)
$$

for $0<a \leq 1$ and $0<p, q<1$.

If we take $a=p_{j}<1$, for all $j \in\{1, \ldots, n\}$, in the above inequalities $\left(a_{1}\right)-\left(c_{1}\right)$ and passing to the sum from 1 to $n$, we deduce the following inequalities $\left(a_{2}\right)-\left(c_{2}\right)$ on $d .(\cdot, \cdot)$.

$\left(a_{2}\right)$

$$
r(q) \sum_{j=1}^{n}\left(\sqrt{p}_{j}-1\right)^{2} \leq \sum_{j=1}^{n} d_{q}\left(p_{j}, 1\right) \leq R(q) \sum_{j=1}^{n}\left(\sqrt{p}_{j}-1\right)^{2}
$$

$\left(b_{2}\right)$

where $0 \leq q \leq 1$.

$$
\frac{1}{2} q(1-q) \sum_{j=1}^{n}\left(p_{j}-1\right)^{2} \leq \sum_{j=1}^{n} d_{q}\left(p_{j}, 1\right) \leq \frac{1}{2} q(1-q) \sum_{j=1}^{n} \frac{\left(p_{j}-1\right)^{2}}{p_{j}}
$$

for $0 \leq q \leq 1$. 
$\left(c_{2}\right)$

$$
\frac{1}{2} q(1-q) \sum_{j=1}^{n} p_{j} \log ^{2} p_{j} \leq \sum_{j=1}^{n} d_{q}\left(p_{j}, 1\right) \leq \frac{1}{2} q(1-q) \sum_{j=1}^{n} \log ^{2} p_{j}
$$

and

$$
\min \left\{\frac{q}{p}, \frac{1-q}{1-p}\right\} \sum_{j=1}^{n} d_{p}\left(p_{j}, 1\right) \leq \sum_{j=1}^{n} d_{q}\left(p_{j}, 1\right) \leq \max \left\{\frac{q}{p}, \frac{1-q}{1-p}\right\} \sum_{j=1}^{n} d_{p}\left(p_{j}, 1\right)
$$

for $0<p, q<1$.

Using the point (i) from Lemma 2 and inequalities $\left(a_{2}\right)-\left(c_{2}\right)$, we deduce a series of inequalities for the Tsallis entropy $H_{q}(\mathbf{p})$ in the following $(\mathrm{A})-(\mathrm{C})$ as the theorem.

Theorem 1. Let $0<p, q<1$. Then we have the following $(A)-(C)$.

(A)

$$
n-1-\frac{R(q)}{1-q} \sum_{j=1}^{n}\left(\sqrt{p}_{j}-1\right)^{2} \leq H_{q}(\mathbf{p}) \leq n-1-\frac{r(q)}{1-q} \sum_{j=1}^{n}\left(\sqrt{p}_{j}-1\right)^{2} .
$$

(B)

$$
n-1-\frac{q}{2} \sum_{j=1}^{n} \frac{\left(p_{j}-1\right)^{2}}{p_{j}} \leq H_{q}(\mathbf{p}) \leq n-1-\frac{q}{2} \sum_{j=1}^{n}\left(p_{j}-1\right)^{2} .
$$

(C)

$$
n-1-\frac{q}{2} \sum_{j=1}^{n} \log ^{2} p_{j} \leq H_{q}(\mathbf{p}) \leq n-1-\frac{q}{2} \sum_{j=1}^{n} p_{j} \log ^{2} p_{j}
$$

and

$$
\begin{aligned}
& (n-1)\left(1-\frac{1-p}{1-q} \max \left\{\frac{q}{p}, \frac{1-q}{1-p}\right\}\right)+\frac{1-p}{1-q} \max \left\{\frac{q}{p}, \frac{1-q}{1-p}\right\} H_{p}(\mathbf{p}) \leq H_{q}(\mathbf{p}) \\
& \leq(n-1)\left(1-\frac{1-p}{1-q} \min \left\{\frac{q}{p}, \frac{1-q}{1-p}\right\}\right)+\frac{1-p}{1-q} \min \left\{\frac{q}{p}, \frac{1-q}{1-p}\right\} H_{p}(\mathbf{p}) .
\end{aligned}
$$

If $p \leq q$, then we have $\min \left\{\frac{q}{p}, \frac{1-q}{1-p}\right\}=\frac{1-q}{1-p}$ and $\max \left\{\frac{q}{p}, \frac{1-q}{1-p}\right\}=\frac{q}{p}$, then we obtain

$$
(n-1) \frac{p-q}{p(1-q)}+\frac{q(1-p)}{p(1-q)} H_{p}(\mathbf{p}) \leq H_{q}(\mathbf{p}) \leq H_{p}(\mathbf{p}),
$$

which implies that $H_{q}(\mathbf{p})$ is decreasing related to $q$.

In the limit of $q \rightarrow 1$, we find some bounds for Shannon entropy as a corollary of the above theorem.

Corollary 1. We have the inequalities for Shannon entropy $H(\mathbf{p})$.

$$
\begin{gathered}
H(\mathbf{p}) \leq n-1-\sum_{j=1}^{n}\left(\sqrt{p_{j}}-1\right)^{2}=2 \sum_{j=1}^{n}\left(\sqrt{p_{j}}-1\right), \\
n-1-\frac{1}{2} \sum_{j=1}^{n} \frac{\left(p_{j}-1\right)^{2}}{p_{j}} \leq H(\mathbf{p}) \leq n-1-\frac{1}{2} \sum_{j=1}^{n}\left(p_{j}-1\right)^{2}, \\
n-1-\frac{1}{2} \sum_{j=1}^{n} \log ^{2} p_{j} \leq H(\mathbf{p}) \leq n-1-\frac{1}{2} \sum_{j=1}^{n} p_{j} \log ^{2} p_{j}
\end{gathered}
$$

and

$$
H(\mathbf{p}) \leq H_{p}(\mathbf{p}), \quad(0<p<1) .
$$


Using the points (ii) and (iii) from Lemma 2 and inequalities $\left(a_{2}\right)-\left(c_{2}\right)$, we deduce several inequalities for Rényi entropy $R_{q}(\mathbf{p})$ and for the Fermi-Dirac-Tsallis entropy $I_{q}^{F D}(\mathbf{p})$ in the following:

Theorem 2. Let $0<q<1$. Then we have

$\left(A_{1}\right)$

$$
\begin{aligned}
& \frac{1}{1-q} \log \left\{n(1-q)+q-R(q)\left(n+1-2 \sum_{j=1}^{n} \sqrt{p}_{j}\right)\right\} \leq R_{q}(\mathbf{p}) \\
& \leq \frac{1}{1-q} \log \left\{n(1-q)+q-r(q)\left(n+1-2 \sum_{j=1}^{n} \sqrt{p}_{j}\right)\right\},
\end{aligned}
$$

$\left(B_{1}\right)$

$$
\begin{aligned}
& \frac{1}{1-q} \log \left\{n(1-q)+q-\frac{1}{2} q(1-q)\left(1-2 n+\sum_{j=1}^{n} \frac{1}{p_{j}}\right)\right\} \leq R_{q}(\mathbf{p}) \\
& \frac{1}{1-q} \log \left\{n(1-q)+q-\frac{1}{2} q(1-q)\left(n-2+\sum_{j=1}^{n} p_{j}^{2}\right)\right\},
\end{aligned}
$$

$\left(C_{1}\right)$

$$
\begin{aligned}
& \frac{1}{1-q} \log \left\{n(1-q)+q-\frac{1}{2} q(1-q) \sum_{j=1}^{n} \log ^{2} p_{j}\right\} \leq R_{q}(\mathbf{p}) \\
& \frac{1}{1-q} \log \left\{n(1-q)+q-\frac{1}{2} q(1-q) \sum_{j=1}^{n} p_{j} \log ^{2} p_{j}\right\},
\end{aligned}
$$

$\left(A_{2}\right)$

$$
\begin{aligned}
& n-\frac{R(q)}{1-q}\left\{3 n-2 \sum_{j=1}^{n}\left(\sqrt{p}_{j}+\sqrt{1-p_{j}}\right)\right\} \leq I_{q}^{F D}(\mathbf{p}) \\
& n-\frac{r(q)}{1-q}\left\{3 n-2 \sum_{j=1}^{n}\left(\sqrt{p}_{j}+\sqrt{1-p_{j}}\right)\right\}
\end{aligned}
$$

$\left(B_{2}\right)$

$$
n-\frac{q}{2}\left(\sum_{j=1}^{n} \frac{1}{p_{j}\left(1-p_{j}\right)}-3 n\right) \leq I_{q}^{F D}(\mathbf{p}) \leq n-\frac{q}{2}\left(n-2+2 \sum_{j=1}^{n} p_{j}^{2}\right)
$$

$\left(C_{2}\right)$

$$
\begin{aligned}
& n-\frac{q}{2} \sum_{j=1}^{n}\left(\log ^{2} p_{j}+\log ^{2}\left(1-p_{j}\right)\right) \leq I_{q}^{F D}(\mathbf{p}) \leq \\
& n-\frac{q}{2} \sum_{j=1}^{n}\left(p_{j} \log ^{2} p_{j}+\left(1-p_{j}\right) \log ^{2}\left(1-p_{j}\right)\right) .
\end{aligned}
$$

In the limit of $q \rightarrow 1$, we find some bounds for the Fermi-Dirac-Tsallis entropy as a corollary of the above theorem. 
Corollary 2. We have the following inequalities for the Fermi-Dirac entropy $I_{1}^{F D}(\mathbf{p})$ :

$$
\begin{aligned}
& I_{1}^{F D}(\mathbf{p}) \leq 2 \sum_{j=1}^{n}\left(\sqrt{p_{j}}+\sqrt{1-p_{j}}-1\right), \\
& \frac{1}{2}\left(5 n-\sum_{j=1}^{n} \frac{1}{p_{j}\left(1-p_{j}\right)}\right) \leq I_{1}^{F D}(\mathbf{p}) \leq \frac{1}{2}\left(n+2-2 \sum_{j=1}^{n} p_{j}^{2}\right)
\end{aligned}
$$

and

$$
\begin{aligned}
& n-\frac{1}{2} \sum_{j=1}^{n}\left(\log ^{2} p_{j}+\log ^{2}\left(1-p_{j}\right)\right) \leq I_{1}^{F D}(\mathbf{p}) \leq \\
& n-\frac{1}{2} \sum_{j=1}^{n}\left(p_{j} \log ^{2} p_{j}+\left(1-p_{j}\right) \log ^{2}\left(1-p_{j}\right)\right) .
\end{aligned}
$$

Theorem 3. Let $0<q<1$. Then,

$\left(A_{3}\right)$

$$
\begin{aligned}
& (2 n+1) r(q)-(n+1) R(q)+2 R(q) \sum_{j=1}^{n} \sqrt{p}_{j}-2 r(q) \sum_{j=1}^{n} \sqrt{1+p_{j}} \\
& \leq(1-q)\left(I_{q}^{B E}(\mathbf{p})-n\right) \\
& \leq(2 n+1) R(q)-(n+1) r(q)+2 r(q) \sum_{j=1}^{n} \sqrt{p}_{j}-2 R(q) \sum_{j=1}^{n} \sqrt{1+p_{j}},
\end{aligned}
$$

$\left(B_{3}\right)$

$$
n+\frac{q}{2}\left(n-\sum_{j=1}^{n} \frac{1}{p_{j}\left(p_{j}+1\right)}\right) \leq I_{q}^{B E}(\mathbf{p}) \leq n+\frac{q}{2}(2-n)
$$

$\left(C_{3}\right)$

$$
\begin{aligned}
& \sum_{j=1}^{n}\left(\log ^{2}\left(p_{j}+1\right)-\log ^{2} p_{j}\right) \\
& \leq \frac{2}{q}\left(I_{q}^{B E}(\mathbf{p})-n\right) \\
& \leq \sum_{j=1}^{n}\left(\left(p_{j}+1\right) \log ^{2}\left(p_{j}+1\right)-p_{j} \log ^{2} p_{j}\right) .
\end{aligned}
$$

Proof. From inequality (7), we find

$$
r(q)\left(\sqrt{p}_{j}-1\right)^{2} \leq d_{q}\left(p_{j}, 1\right) \leq R(q)\left(\sqrt{p}_{j}-1\right)^{2}
$$

and

$$
r(q)\left(\sqrt{p_{j}+1}-1\right)^{2} \leq d_{q}\left(p_{j}+1,1\right) \leq R(q)\left(\sqrt{p_{j}+1}-1\right)^{2} .
$$

Using inequalities (19), (20) and the definition of the Bose-Einstein-Tsallis entropy $I_{q}^{B E}(\mathbf{p})$, given above, we find

$$
n+\frac{1}{1-q}\left(r(q) \sum_{j=1}^{n}\left(\sqrt{p_{j}+1}-1\right)^{2}-R(q) \sum_{j=1}^{n}\left(\sqrt{p}_{j}-1\right)^{2}\right) \leq I_{q}^{B E}(\mathbf{p})
$$




$$
\leq n+\frac{1}{1-q}\left(R(q) \sum_{j=1}^{n}\left(\sqrt{p_{j}+1}-1\right)^{2}-r(q) \sum_{j=1}^{n}\left(\sqrt{p}_{j}-1\right)^{2}\right),
$$

which implies inequality (16). From inequality (8), we have:

$$
\frac{1}{2} q(1-q) \frac{p_{j}^{2}}{p_{j}+1} \leq d_{q}\left(p_{j}+1,1\right) \leq \frac{1}{2} q(1-q) p_{j}^{2}
$$

and

$$
\frac{1}{2} q(1-q)\left(p_{j}-1\right)^{2} \leq d_{q}\left(p_{j}, 1\right) \leq \frac{1}{2} q(1-q) \frac{\left(p_{j}-1\right)^{2}}{p_{j}} .
$$

Summing from 1 to $n$, we deduce inequality (17).

We apply inequality (9) in the following way:

$$
\frac{1}{2} q(1-q) \log ^{2}\left(p_{j}+1\right) \leq d_{q}\left(p_{j}+1,1\right) \leq \frac{1}{2} q(1-q)\left(p_{j}+1\right) \log ^{2}\left(p_{j}+1\right)
$$

and

$$
\frac{1}{2} q(1-q) p_{j} \log ^{2} p_{j} \leq d_{q}\left(p_{j}, 1\right) \leq \frac{1}{2} q(1-q) p_{j} \log ^{2} p_{j} .
$$

Summing from 1 to $n$, we deduce inequality (18).

Corollary 3. We have the following inequalities for the Bose-Einstein entropy $I_{1}^{B E}(\mathbf{p})$ :

$$
3 n-\sum_{j=1}^{n} \frac{1}{p_{j}\left(1+p_{j}\right)} \leq 2 I_{1}^{B E}(\mathbf{p}) \leq n+1
$$

and

$$
\sum_{j=1}^{n}\left(\log ^{2}\left(p_{j}+1\right)-\log ^{2} p_{j}\right) \leq 2\left(I_{1}^{B E}(\mathbf{p})-n\right) \leq \sum_{j=1}^{n}\left(\left(p_{j}+1\right) \log ^{2}\left(p_{j}+1\right)-p_{j} \log ^{2} p_{j}\right) .
$$

\section{New Characterizations of Young's Inequality}

The inequality of Young is given by:

$$
p a+(1-p) b \geq a^{p} b^{1-p}, \quad(a, b>0, \quad p \in[0,1]),
$$

which means $d_{p}(a, b) \geq 0$.

In this section, we give further bounds on $d .(\cdot, \cdot)$.

Lemma 3. Let $a$ and $b$ be positive real numbers, and let $p \in \mathbb{R}$. Then,

$$
d_{p}(a, b)=p \sum_{k=1}^{n} 2^{k-1} \sqrt[2^{k-1}]{b^{2^{k-1}-1}}\left(\sqrt[2^{k}]{a}-\sqrt[2^{k}]{b}\right)^{2}+d_{2^{n}} p\left(\sqrt[2^{n}]{a b^{2^{n}}-1}, b\right)
$$

and

$$
d_{p}(a, b)=(1-p) \sum_{k=1}^{n} 2^{k-1} \sqrt[2^{k-1}]{a^{2^{k-1}-1}}\left(\sqrt[2^{k}]{a}-\sqrt[2^{k}]{b}\right)^{2}+d_{2^{n}(p-1)+1}\left(a, \sqrt[2^{n}]{a^{2^{n}-1} b}\right)
$$

Proof. Using Lemma 2 for $p \in \mathbb{R}$, then

$$
d_{p}(a, b)=p(\sqrt{a}-\sqrt{b})^{2}+d_{2 p}(\sqrt{a b}, b) .
$$


We replace $p$ by $2 p$ and $a$ by $\sqrt{a b}$, then we get

$$
d_{2 p}(\sqrt{a b}, b)=2 p(\sqrt[4]{a b}-\sqrt{b})^{2}+d_{4 p}\left(\sqrt[4]{a b^{3}}, b\right) .
$$

If we inductively repeat the above substitutions, for $k \geq 1$, then we have

$$
d_{2^{k-1} p}\left(\sqrt[2^{k-1}]{a b^{2^{k-1}-1}}, b\right)=2^{k-1} p\left(\sqrt[2^{k}]{a b^{2^{k-1}-1}}-\sqrt{b}\right)^{2}+d_{2^{k}}\left(\sqrt[2^{k}]{a b^{2^{k}-1}}, b\right) .
$$

Therefore, summarizing the above relations for $k \in\{1, \ldots, n\}$, we obtain the relation of the statement. Applying equality (21) and taking into account that $d_{p}(a, b)=d_{1-p}(b, a)$, we deduce equality (22).

Remark 1. From [8], if $a, b>0$ and $p \notin[0,1]$, we have $d_{1-p}(a, b) \leq 0$, so, we deduce $d_{2^{n} p}\left(\sqrt[2^{n}]{a b^{2^{n}}-1}, b\right) \leq 0$, for $p \notin\left[0, \frac{1}{2^{n}}\right]$ and $d_{2^{n}(p-1)+1}\left(a, \sqrt[2^{n}]{a^{2^{n}}-1 b}\right) \leq 0$, for $p \notin\left[1-\frac{1}{2^{n}}, 1\right]$. Using the above equalities, we deduce the inequalities:

$$
d_{p}(a, b) \leq p \sum_{k=1}^{n} 2^{k-1} \sqrt[2^{k-1}]{b^{2^{k-1}-1}}\left(\sqrt[2^{k}]{a}-\sqrt[2^{k}]{b}\right)^{2}
$$

when $p \notin\left[0, \frac{1}{2^{n}}\right]$ and

$$
d_{p}(a, b) \leq(1-p) \sum_{k=1}^{n} 2^{k-1} \sqrt[2^{k-1}]{a^{2^{k-1}-1}}\left(\sqrt[2^{k}]{a}-\sqrt[2^{k}]{b}\right)^{2} .
$$

when $p \notin\left[1-\frac{1}{2^{n}}, 1\right]$. These inequalities are given by Furuichi et al. in ([15], Theorem 3). We also find that inequality (23) when $p \leq 0$ and inequality (24) when $p \geq 1$ are given by Sababheh-Choi in ([8], Theorem 2.9) and by Sababheh-Moslehian ([16], Theorem 2.2).

Proposition 1. Let $a$ and $b$ be positive real numbers. We then have the following bounds on $d .(\cdot, \cdot)$.

(i) For $p \in\left[0, \frac{1}{2^{n}}\right]$, we have

$$
\begin{aligned}
& r\left(2^{n} p\right)\left(\sqrt[2^{n+1}]{a b^{2^{n}-1}}-\sqrt{b}\right)^{2}+p \sum_{k=1}^{n} \sqrt[2^{k-1}]{b^{2^{k-1}-1}}\left(\sqrt[2^{k}]{a}-\sqrt[2^{k}]{b}\right)^{2} \leq d_{p}(a, b) \\
& \leq R\left(2^{n} p\right)\left(\sqrt[2^{n+1}]{a b^{2^{n}}-1}-\sqrt{b}\right)^{2}+p \sum_{k=1}^{n} \sqrt[2^{k-1}]{b^{2^{k-1}-1}}\left(\sqrt[2^{k}]{a}-\sqrt[2^{k}]{b}\right)^{2}
\end{aligned}
$$

where $r(\cdot)$ and $R(\cdot)$ are defined above,

(ii) For $p \in\left[0, \frac{1}{2^{n}}\right]$, we have

$$
\begin{gathered}
2^{n-1} p\left(1-2^{n} p\right) \frac{\left(\sqrt[2^{n}]{a b^{2^{n}}-1}-b\right)^{2}}{\max \left\{\sqrt[2^{n}]{a b^{2^{n}}-1}, b\right\}}+p \sum_{k=1}^{n} \sqrt[2^{k-1}]{b^{2^{k-1}-1}}\left(\sqrt[2^{k}]{a}-\sqrt[2^{k}]{b}\right)^{2} \leq d_{p}(a, b) \\
2^{n-1} p\left(1-2^{n} p\right) \frac{\left(\sqrt[2^{n}]{a b^{2^{n}}-1}-b\right)^{2}}{\min \left\{\sqrt[2^{n}]{a b^{2^{n}}-1}, b\right\}}+p \sum_{k=1}^{n} \sqrt[2^{k-1}]{b^{2^{k-1}-1}}\left(\sqrt[2^{k}]{a}-\sqrt[2^{k}]{b}\right)^{2} .
\end{gathered}
$$


(iii) For $p \in\left[0, \frac{1}{2^{n}}\right]$, we have

$$
\begin{aligned}
& \frac{p}{2^{n+1}}\left(1-2^{n} p\right) \min \left\{\sqrt[2^{n}]{a b^{2^{n}-1}}, b\right\} \log ^{2} \frac{a}{b} \\
& \leq d_{p}(a, b)-p \sum_{k=1}^{n} \sqrt[2^{k-1}]{b^{2^{k-1}-1}}\left(\sqrt[2^{k}]{a}-\sqrt[2^{k}]{b}\right)^{2} \\
& \leq \frac{p}{2^{n+1}}\left(1-2^{n} p\right) \min \left\{\sqrt[2^{n}]{a b^{2^{n}-1}}, b\right\} \log ^{2} \frac{a}{b}
\end{aligned}
$$

Proof. We use the inequalities from (a) to (c), where we replace $p$ by $2^{n} p$ and $a$ by $\sqrt[2^{n}]{a b^{2^{n}}-1}$. For $a, b>0$ and $p \in\left[0, \frac{1}{2^{n}}\right]$, we have the following $\left(a_{3}\right)-\left(c_{3}\right)$.

$\left(a_{3}\right)$

$$
\begin{aligned}
& r\left(2^{n} p\right)\left(\sqrt[2^{n+1}]{a b^{2^{n}}-1}-\sqrt{b}\right)^{2} \leq d_{2^{n}} p\left(\sqrt[2^{n}]{a b^{2^{n}-1}}, b\right) \\
& \leq R\left(2^{n} p\right)\left(\sqrt[2^{n+1}]{a b^{2^{n}}-1}-\sqrt{b}\right)^{2}
\end{aligned}
$$

$\left(b_{3}\right)$

$$
\begin{aligned}
& 2^{n-1} p\left(1-2^{n} p\right) \frac{\left(\sqrt[2^{n}]{a b^{2^{n}}-1}-b\right)^{2}}{\max \left\{\sqrt[2^{n}]{a b^{2^{n}}-1}, b\right\}} \leq d_{2^{n} p}\left(\sqrt[2^{n}]{a b^{2^{n}}-1}, b\right) \\
& \leq 2^{n-1} p\left(1-2^{n} p\right) \frac{\left(\sqrt[2^{n}]{a b^{2^{n}}-1}-b\right)^{2}}{\min \left\{\sqrt[2^{n}]{a b^{2^{n}}-1}, b\right\}}
\end{aligned}
$$

$\left(c_{3}\right)$

$$
\begin{aligned}
& \frac{p}{2^{n+1}}\left(1-2^{n} p\right) \min \left\{\sqrt[2^{n}]{a b^{2^{n}}-1}, b\right\} \log ^{2} \frac{a}{b} \leq d_{2^{n} p}\left(\sqrt[2^{n}]{a b^{2^{n}-1}}, b\right) \\
& \leq \frac{p}{2^{n+1}}\left(1-2^{n} p\right) \max \left\{\sqrt[2^{n}]{a b^{2^{n}}-1}, b\right\} \log ^{2} \frac{a}{b} .
\end{aligned}
$$

Using equality (21) and inequalities (26)-(28), we deduce the inequalities from the statement.

\section{The Connection between $d .(\cdot, \cdot)$ and Different Types of Convexity}

In the following, we use the inequality by Kittaneh-Manasrah as noted in (3). We prepare some lemmas to state our results.

Lemma 4. If $f: J \rightarrow \mathbb{R}$, where $J$ is an interval of $\mathbb{R}$, is a concave function, then

$$
f((1+r) x-r y) \leq(1+r) f(x)-r f(y)
$$

for all $x, y \in J$ and all $r>0$ with $(1+r) x-r y \in J$. If $f$ is a convex function, then the reversed inequality above holds.

Proof. If $f$ is concave, then we have

$$
\frac{1}{1+r} f((1+r) x-r y)+\frac{r}{1+r} f(y) \leq f\left(x-\frac{r}{1+r} y+\frac{r}{1+r} y\right)=f(x) .
$$


The following result is given in ([15], Corollary 1). This is the supplemental to the first inequality of (3).

Lemma 5. Let $a$ and $b$ be positive real numbers and let $p \notin(0,1)$. Then,

$$
d_{p}(a, b) \leq r(p)(\sqrt{a}-\sqrt{b})^{2}
$$

where $r(p):=\min \{p, 1-p\}$

Proof. We set the function $f(t):=t^{p}-2 p t^{1 / 2}-(1-2 p)$ for $t>0$ and $p \notin(0,1 / 2)$. From $f^{\prime}(t)=p t^{-1 / 2}\left(t^{p-1 / 2}-1\right)$, we find that $f^{\prime}(t)=0 \Leftrightarrow t=1, f^{\prime}(t)<0$ for $0<t<1$ and $f^{\prime}(t)>0$ for $t>1$. Thus, we have $f(t) \geq f(1)=0$. Putting $t:=a / b$ and multiplying $b>0$ to both sides in the inequality $f(t) \geq 0$, we have

$$
a^{p} b^{1-p} \geq 2 p \sqrt{a b}+(1-2 p) b
$$

which is equivalent to

$$
p a+(1-p) b-p(\sqrt{a}-\sqrt{b})^{2} \leq a^{p} b^{1-p}, \quad p \notin(0,1 / 2)
$$

We similarly have

$$
p a+(1-p) b-(1-p)(\sqrt{a}-\sqrt{b})^{2} \leq a^{p} b^{1-p}, \quad p \notin(1 / 2,1) .
$$

From (31) and (32), we have (30).

Note that the supplemental to the second inequality of (3), never generally holds:

$$
R(p)(\sqrt{a}-\sqrt{b})^{2} \leq d_{p}(a, b), \quad a, b>0 \quad p \notin(0,1) .
$$

To state the following result, we review the log-convexity/log-concavity. For the function $f: I \rightarrow(0, \infty)$, where $I \subset \mathbb{R}, x, y \in I$ and $\lambda \in[0,1]$, if $f((1-\lambda) x+\lambda y) \leq$ $f^{1-\lambda}(x) f^{\lambda}(y)$, then $f$ is often called log-convex function. If the reversed inequality holds, then $f$ is called log-concave function.

In the following two lemmas, we deal with the symmetric function on $\frac{1}{2}$ (i.e., $f(t)=$ $f(1-t)$, for every $t \in[0,1])$. The results are applied to the concrete symmetric function related to entropy, in the end of this section.

Lemma 6. Let $f:[0,1] \rightarrow(0, \infty)$ be a convex function such that $f(t)=f(1-t)$ for every $t \in[0,1]$. Then

$$
2 R(t) f(1 / 2)+(1-2 R(t)) f(0) \leq f(t) \leq 2 r(t) f(1 / 2)+(1-2 r(t)) f(0),
$$


where $r(t):=\min \{t, 1-t\}$ and $R(t):=\max \{t, 1-t\}$. If in addition, $f$ is log-convex, then

$$
\begin{aligned}
& 2 R(t) f(1 / 2)+(1-2 R(t)) f(0) \\
& \leq 2 R(t) f(1 / 2)+(1-2 R(t)) f(0)-(1-2 R(t))(\sqrt{f(1 / 2)}-\sqrt{f(0)})^{2} \\
& \leq f(1 / 2)^{2 R(t)} f(0)^{1-2 R(t)} \\
& \leq f(t) \\
& \leq f(1 / 2)^{2 r(t)} f(0)^{1-2 r(t)} \\
& \leq 2 r(t) f(1 / 2)+(1-2 r(t)) f(0)-r(2 r(t))(\sqrt{f(1 / 2)}-\sqrt{f(0)})^{2} \\
& \leq 2 r(t) f(1 / 2)+(1-2 r(t)) f(0) .
\end{aligned}
$$

Proof. By convexity of $f$, we have for $t \in[0,1 / 2]$,

$$
f(t)=f\left(2 t \cdot \frac{1}{2}+(1-2 t) \cdot 0\right) \leq 2 t f\left(\frac{1}{2}\right)+(1-2 t) f(0) .
$$

Thus, we have

$$
2 t(f(1 / 2)-f(0)) \geq f(t)-f(0) .
$$

For $t \in[1 / 2,1]$, by exchanging $t$ with $1-t$ in the above inequality, we have

$$
2(1-t)(f(1 / 2)-f(0)) \geq f(t)-f(0) .
$$

Therefore, we have

$$
2 r(t)(f(1 / 2)-f(0)) \geq f(t)-f(0),
$$

which implies the second inequality in (33). By Lemma 4 with $r:=2 t-1>0$ (i.e., $t \in[1 / 2,1]$.$) , we have$

$$
\begin{aligned}
f(t) & =f\left(2 t \cdot \frac{1}{2}+(1-2 t) \cdot 0\right)=f\left((1+r) \cdot \frac{1}{2}-r \cdot 0\right) \\
& \geq(1+r) f(1 / 2)-r f(0)=2 t f(1 / 2)+(1-2 t) f(0) .
\end{aligned}
$$

Thus, we have for $t \in[1 / 2,1]$

$$
2 t(f(1 / 2)-f(0)) \leq f(t)-f(0) .
$$

For $t \in[0,1 / 2]$, by exchanging $t$ with $1-t$ in the above inequality, we have

$$
2(1-t)(f(1 / 2)-f(0)) \leq f(t)-f(0) .
$$

Therefore, we have

$$
2 R(t)(f(1 / 2)-f(0)) \leq f(t)-f(0),
$$

which implies the first inequality in (33).

By log-convexity of $f, \log f$ is convex so that we have $f(t) \leq f(1 / 2)^{2 r(t)} f(0)^{1-2 r(t)}$ which is the forth inequality of (34). The third inequality is from (33) and the second one is 
obtained by the Young inequality. The last inequality of (34) is trivial. Since $0 \leq r(t) \leq \frac{1}{2}$, we have $0 \leq 2 r(t) \leq 1$. So we can use the first inequality of (3) as

$$
\begin{aligned}
& f(1 / 2)^{2 r(t)} f(0)^{1-2 r(t)} \\
& \leq 2 r(t) f(1 / 2)+(1-2 r(t)) f(0)-r(2 r(t))(\sqrt{f(1 / 2)}-\sqrt{f(0)})^{2},
\end{aligned}
$$

which is the fifth inequality of (34). Finally, we prove the first inequality of (34). Since $\frac{1}{2} \leq R(t) \leq 1$, we have $1 \leq 2 R(t) \leq 2$ and $-3 \leq 1-2 R(t) \leq-1$. Namely, we have $1-2 R(t) \leq 2 R(t)$. By using (30), we have

$$
\begin{aligned}
& f(1 / 2)^{2 R(t)} f(0)^{1-2 R(t)} \\
& \geq 2 R(t) f(1 / 2)+(1-2 R(t)) f(0)-(1-2 R(t))(\sqrt{f(1 / 2)}-\sqrt{f(0)})^{2} .
\end{aligned}
$$

It is notable that the right inequalities in (33) and (34) are also found in ([17], Lemma 1.1). The following lemma is a counterpart by concavity. However, it does not completely corresponded to the above lemma. (See Remark 2 below).

Lemma 7. Let $f:[0,1] \rightarrow(0, \infty)$ be a concave function with $f(t)=f(1-t)$ for every $t \in[0,1]$. Then

$$
2 r(t) f(1 / 2)+(1-2 r(t)) f(0) \leq f(t) \leq 2 R(t) f(1 / 2)+(1-2 R(t)) f(0) .
$$

If in addition, $f$ is log-concave, then

$$
\begin{aligned}
& 2 r(t) f(1 / 2)+(1-2 r(t)) f(0)-R(2 r(t))(\sqrt{f(1 / 2)}-\sqrt{f(0)})^{2} \\
& \leq f(1 / 2)^{2 r(t)} f(0)^{1-2 r(t)} \\
& \leq 2 r(t) f(1 / 2)+(1-2 r(t)) f(0) \\
& \leq f(t) \\
& \leq 2 R(t) f(1 / 2)+(1-2 R(t)) f(0) \\
& \leq f(1 / 2)^{2 R(t)} f(0)^{1-2 R(t)}
\end{aligned}
$$

Proof. By concavity of $f$, we have for $t \in[0,1 / 2]$,

$$
f(t)=f\left(2 t \cdot \frac{1}{2}+(1-2 t) \cdot 0\right) \geq 2 t f(1 / 2)+(1-2 t) f(0),
$$

which implies

$$
2 t(f(0)-f(1 / 2)) \geq f(0)-f(t) .
$$

For the case of $t \in[1 / 2,1]$, by exchanging $t$ with $1-t$, then we have from the above inequality

$$
2(1-t)(f(0)-f(1 / 2)) \geq f(0)-f(1-t)=f(0)-f(t) .
$$

Thus, we have for $t \in[0,1]$ and $r(t):=\min \{t, 1-t\}$,

$$
2 r(t)(f(0)-f(1 / 2)) \geq f(0)-f(t),
$$


which implies the first inequality of (35). For the proof of the second inequality of (35), we use Lemma 4. Putting $r:=2 t-1>0$ in (29), we have

$$
\begin{aligned}
f(t) & =f\left(2 t \cdot \frac{1}{2}+(1-2 t) \cdot 0\right)=f\left((1+r) \cdot \frac{1}{2}-r \cdot 0\right) \leq(1+r) f(1 / 2)-r f(0) \\
& =2 t f(1 / 2)+(1-2 t) f(0),
\end{aligned}
$$

which means

$$
2 t(f(0)-f(1 / 2)) \leq f(0)-f(t), t \in[1 / 2,1] .
$$

For the case of $t \in[0,1 / 2]$, by exchanging $t$ with $1-t$, we have from the above inequality

$$
2(1-t)(f(0)-f(1 / 2)) \leq f(0)-f(1-t), t \in[0,1 / 2] .
$$

By the symmetric property of $f$ in $t=1 / 2$, we obtain

$$
2 R(t)(f(0)-f(1 / 2)) \leq f(0)-f(t),
$$

which gives the right hand side in the inequalities (35).

If $f$ is log-concave, then we have from the first inequality of (35) with concave function $\log f, f(1 / 2)^{2 R(t)} f(0)^{1-2 R(t)} \geq f(t)$, which show the forth inequality in (36). The third inequality is just from (35). The second and last inequalities in (36) are obtained by the Young inequality.

Since we have $0 \leq r(t) \leq 1 / 2 \leq R(t) \leq 1$ generally, we have $0 \leq 2 r(t) \leq 1$ for $t \in[0,1]$. Then we apply the second inequality of (3), we have

$$
f(1 / 2)^{2 r(t)} f(0)^{1-2 r(t)} \geq 2 r(t) f(1 / 2)+(1-2 r(t)) f(0)-R(2 r(t))(\sqrt{f(1 / 2)}-\sqrt{f(0)})^{2}
$$

which shows the first inequality in (36).

Remark 2. In general, we have the supplement to the Young inequality:

$$
a^{v} b^{1-v} \geq v a+(1-v) b, \quad v \notin(0,1), \quad a, b>0 .
$$

Thus, we have

$$
f(1 / 2)^{2 R(t)} f(0)^{1-2 R(t)} \geq 2 R(t) f(1 / 2)+(1-2 R(t)) f(0) .
$$

Therefore, it seems difficult to bound $f(1 / 2)^{2 R(t)} f(0)^{1-2 R(t)}$ in (36) from the above by the use of the two terms $2 R(t) f(1 / 2)+(1-2 R(t)) f(0)$ and $(\sqrt{f(1 / 2)}-\sqrt{f(0)})^{2}$ as a simple form.

We have some bounds on $f(1 / 2)^{2 R(t)} f(0)^{1-2 R(t)}$ by applying (3)-(6). We here show one result by the use of (3). However, we omit the other cases.

Lemma 8. Let $a$ and $b$ be positive real numbers and let $p \in[1,2]$. Then,

$$
\begin{aligned}
& p a+(1-p) b+\min \left\{A_{p}, B_{p}\right\}(\sqrt{a}-\sqrt{b})^{2} \\
& \leq a^{p} b^{1-p} \\
& \leq p a+(1-p) b+\max \left\{A_{p}, B_{p}\right\}(\sqrt{a}-\sqrt{b})^{2},
\end{aligned}
$$

where $A_{p}:=(p-1)\left(1+2 \sqrt{\frac{a}{b}}\right)$ and $B_{p}:=(2 p-3) \frac{a}{b}+(p-1)\left(1+2 \sqrt{\frac{a}{b}}\right)$.

Proof. Since $p-1 \in[0,1]$, we can use (3) as 


$$
r(p-1)(\sqrt{a}-\sqrt{b})^{2} \leq d_{p-1}(a, b) \leq R(p-1)(\sqrt{a}-\sqrt{b})^{2} .
$$

Here we have the relation:

$$
b \cdot d_{p}(a, b)-a \cdot d_{p-1}(a, b)=(1-p)(a-b)^{2}, \quad(a, b>0, \quad p \in \mathbb{R}) .
$$

Combining (39) with (38), we obtain

$$
\begin{aligned}
& r(p-1) a(\sqrt{a}-\sqrt{b})^{2}+(1-p)(a-b)^{2} \leq b \cdot d_{p}(a, b) \\
& \leq R(p-1) a(\sqrt{a}-\sqrt{b})^{2}+(1-p)(a-b)^{2} .
\end{aligned}
$$

Elementary calculations imply

$$
\begin{aligned}
& p a+(1-p) b+\frac{1}{b}\left\{(p-1)(\sqrt{a}+\sqrt{b})^{2}-R(p-1) a\right\}(\sqrt{a}-\sqrt{b})^{2} \\
& \leq a^{p} b^{1-p} \\
& \leq p a+(1-p) b+\frac{1}{b}\left\{(p-1)(\sqrt{a}+\sqrt{b})^{2}-r(p-1) a\right\}(\sqrt{a}-\sqrt{b})^{2} .
\end{aligned}
$$
ities (37).

As for the bounds on $f(1 / 2)^{2 R(t)} f(0)^{1-2 R(t)}$, we have the following result.

Proposition 2. Let $t \in[0,1]$ and a function $f:[0,1] \rightarrow(0, \infty)$. Then we have

$$
\begin{aligned}
& 2 R(t) f(1 / 2)+(1-2 R(t)) f(0)+\min \left\{A_{t}, B_{t}\right\}(\sqrt{f(1 / 2)}-\sqrt{f(0)})^{2} \\
& \leq f(1 / 2)^{2 R(t)} f(0)^{1-2 R(t)} \\
& \leq 2 R(t) f(1 / 2)+(1-2 R(t)) f(0)+\max \left\{A_{t}, B_{t}\right\}(\sqrt{f(1 / 2)}-\sqrt{f(0)})^{2},
\end{aligned}
$$

where

$$
\begin{aligned}
& A_{t}:=(2 R(t)-1)\left(1+2 \sqrt{\frac{f(1 / 2)}{f(0)}}\right), \\
& B_{t}:=(4 R(t)-3) \frac{f(1 / 2)}{f(0)}+(2 R(t)-1)\left(1+2 \sqrt{\frac{f(1 / 2)}{f(0)}}\right) .
\end{aligned}
$$

Proof. Since $1 \leq 2 R(t) \leq 2$, we set $p:=2 R(t), a:=f(1 / 2)$ and $b:=f(0)$ in Lemma 8 .

Example 1. The so-called binary entropy (e.g., [18], example 2.1.1) defined by

$$
h_{b i n}(t):=-t \log t-(1-t) \log (1-t)>0,(0<t<1)
$$

with convention $0 \log 0=: 0$, satisfies the conditions in Lemma 7, since

$$
\frac{d^{2} h_{b i n}(t)}{d t^{2}}=\frac{-1}{t(1-t)}<0
$$

and

$$
\frac{d^{2}}{d t^{2}}\left(\log h_{b i n}(t)\right)=\frac{-h_{b i n}(t)-t(1-t)\{\log t-\log (1-t)\}^{2}}{t(1-t) h_{b i n}(t)^{2}}<0
$$


The standard convention $0 \log 0=: 0$ is in information theory, since we have $\lim _{x \downarrow 0} x \log x=0$ and $\log x$ is undefined for $x \leq 0$. In information theory, we use 2 as the base of the logarithmic function, but we here use e for mathematical simplicity. Its selection is not essential in mathematics. Applying (35) to function $h_{\text {bin }}(t)$ with convention $h_{\text {bin }}(0)=: 0$, we have $2\left(\log _{e} 2\right) r(t) \leq h_{\text {bin }}(t) \leq 2\left(\log _{e} 2\right) R(t)$, which is equivalent to

$$
2 \min \{t, 1-t\} \leq-t \log _{2} t-(1-t) \log _{2}(1-t) \leq 2 \max \{t, 1-t\} .
$$

The above inequalities are equivalent to

$$
1-|1-2 p| \leq H_{b}(p) \leq 1+|1-2 p|,(0 \leq p \leq 1),
$$

where $H_{b}(p):=-p \log _{2} p-(1-p) \log _{2}(1-p)$ is the usual binary entropy, whose base is 2 .

If we do not adopt the standard convention $0 \log 0=: 0$ in information theory, then we assume $f(0):=\lim _{t \rightarrow 0} f(t)=: \varepsilon$ precisely. Applying the inequalities in (36):

$$
f(1 / 2)^{2 r(t)} f(0)^{1-2 r(t)} \leq f(t) \leq f(1 / 2)^{2 R(t)} f(0)^{1-2 R(t)},
$$

we obtain

$$
\left(\log _{e} 2\right)^{2 r(t)} \varepsilon^{1-2 r(t)} \leq f(t) \leq\left(\log _{e} 2\right)^{2 R(t)} \varepsilon^{1-2 R(t)} ，
$$

which implies the following result.

$$
\left(\frac{\varepsilon}{\log _{e} 2}\right)^{1-2 r(p)} \leq H_{b}(p) \leq\left(\frac{\varepsilon}{\log _{e} 2}\right)^{1-2 R(p)}, \quad(0 \leq p \leq 1) .
$$

The Fermi-Dirac entropy is defined above by

$$
I_{1}^{F D}(\mathbf{p}):=-\sum_{j=1}^{n} p_{j} \log p_{j}-\sum_{j=1}^{n}\left(1-p_{j}\right) \log \left(1-p_{j}\right) .
$$

From the bounds of the binary entropy given in (40) and (41), we obtain the interesting bounds on the Fermi-Dirac entropy as

$$
2 \sum_{j=1}^{n} \min \left\{p_{j}, 1-p_{j}\right\} \leq I_{1}^{F D}(\mathbf{p}) \leq 2 \sum_{j=1}^{n} \max \left\{p_{j}, 1-p_{j}\right\}
$$

or

$$
n-\sum_{j=1}^{n}\left|1-2 p_{j}\right| \leq I_{1}^{F D}(\mathbf{p}) \leq n+\sum_{j=1}^{n}\left|1-2 p_{j}\right| .
$$

\section{Concluding Remarks}

We close this paper by providing some remarks on the log-convex function.

Lemma 9. For $a, b, c, d>0$ and $\lambda \in[0,1]$, we have

$$
a^{\lambda} b^{1-\lambda}+c^{\lambda} d^{1-\lambda} \leq(a+c)^{\lambda}(b+d)^{1-\lambda} .
$$

Proof. Since function $f(t)=t^{\lambda}$ is concave for $\lambda \in[0,1]$, we use the Jensen inequality for positive real numbers $x$ and $y$ as

$$
\frac{b f(x)+d f(y)}{b+d} \leq f\left(\frac{b x+d y}{b+d}\right) .
$$


If we take $x:=\frac{a}{b}$ and $y:=\frac{c}{d}$, then we obtain

$$
\frac{b}{b+d}\left(\frac{a}{b}\right)^{\lambda}+\frac{d}{b+d}\left(\frac{c}{d}\right)^{\lambda} \leq\left(\frac{a+c}{b+d}\right)^{\lambda}
$$

which implies (42).

Theorem 4. If $f, g: I \rightarrow(0, \infty)$ are log-convex functions, then function $\mu f+v g$ is log-convex, where $I \subset \mathbb{R}$ and $\mu, v>0$.

Proof. Since $f, g$ are log-convex functions, we have for $\lambda \in[0,1]$,

$$
\begin{aligned}
& (\mu f+v g)(\lambda x+(1-\lambda) y)=\mu f(\lambda x+(1-\lambda) y)+v g(\lambda x+(1-\lambda) y) \\
& \leq \mu f^{\lambda}(x) f^{1-\lambda}(y)+v g^{\lambda}(x) g^{1-\lambda}(y)=(\mu f(x))^{\lambda}(\mu f(y))^{1-\lambda}+(v g(x))^{\lambda}(v g(y))^{1-\lambda} \\
& \leq(\mu f(x)+v g(x))^{\lambda}(\mu f(y)+v g(y))^{1-\lambda},
\end{aligned}
$$

where we used Lemma 9 in the last inequality. Therefore, $\mu f+v g$ is log-convex.

Let $\mathbb{M}_{n}$ be the set of all $n \times n$ complex matrices, and let $\mathbb{M}_{n}^{+}$be the set of all positive semi-definite matrices in $\mathbb{M}_{n}$.

Corollary 4. For $A, B \in \mathbb{M}_{n}^{+}, X \in \mathbb{M}_{n}, t \in[0,1]$ and $\||\cdot|\|$ is the unitarily invariant norm, the following functions are log-convex:

$$
\begin{aligned}
& g_{1}(t):=\left\|\left|A^{t} X B^{t}\right|\right\|+\left\|\left|A^{1-t} X B^{1-t}\right|\right\|, \\
& g_{2}(t):=\left\||| A^{t} X B^{1-t}|\|+\|| \mid A^{1-t} X B^{t}\right\|, \\
& g_{3}(t):=\|\| A^{t}|\|+\||\left|A^{1-t}\right| \|, \\
& g_{4}(t):=\operatorname{tr}\left(A^{t} X B^{1-t} X^{*}+A^{1-t} X B^{t} X^{*}\right) .
\end{aligned}
$$

Proof. In [19], it was shown that functions $f_{1}(t):=|| A^{t} X B^{t}\left|\|, f_{2}(t):=\right|\left|A^{t} X B^{1-t}\right| \mid$, $f_{3}(t):=\left|\| A^{t}\right|||$ and $f_{4}(t):=\operatorname{tr}\left(A^{t} X B^{1-t} X^{*}\right)$ are log-convex on $[0,1]$. Thus, we have the corollary from Theorem 4 .

Since the functions $g_{i}$ are log-convex and $g_{i}(t)=g_{i}(1-t)$, we can apply Lemma 6 for the symmetric function $g_{i}$ on an axis $t=\frac{1}{2}$. Therefore, we obtain the chain of inequalities for the functions $g_{1}$ in the following, for example. We can obtain the similar inequalities for the other functions $g_{2}, g_{3}$ and $g_{4}$. However, we omit them. For $A, B \in \mathbb{M}_{n}^{+}, X \in \mathbb{M}_{n}$ and $t \in[0,1]$, we have

$$
\begin{aligned}
& 4 R(t) \|\left|A^{1 / 2} X B^{1 / 2}\right|||+(1-2 R(t))(|||X|||+|||A X B|||) \\
& -(1-2 R(t))\left(\sqrt{2\left|\| A^{1 / 2} X B^{1 / 2}\right| \mid}-\sqrt{\||| X|\|+|\|A X B \mid\|}\right)^{2} \\
& \leq\left(2||\left|A^{1 / 2} X B^{1 / 2}\right|||\right)^{2 R(t)}(|||X|||+|||A X B|||)^{1-2 R(t)} \\
& \leq \||| A^{t} X B^{t}|||+||| A^{1-t} X B^{1-t}|| \mid \\
& \leq\left(2||\left|A^{1 / 2} X B^{1 / 2}\right|||\right)^{2 r(t)}(|| X|\||+|||A X B|||)^{1-2 r(t)} \\
& \leq 4 r(t)||\left|A^{1 / 2} X B^{1 / 2}\right|||+(1-2 r(t))(|\| X|||+|||A X B|||) \\
& -r(2 r(t))\left(\sqrt{2||\left|A^{1 / 2} X B^{1 / 2}\right| \mid}-\sqrt{|\|X|\|+||| A X B|| \mid}\right)^{2} .
\end{aligned}
$$


Author Contributions: This work was carried out in collaboration among all authors. All authors contributed equally and significantly in writing this manuscript. All authors have read and agreed to the published version of the manuscript.

Funding: The first author was supported in part by JSPS KAKENHI grant number 21K03341.

Institutional Review Board Statement: Not applicable.

Informed Consent Statement: Not applicable.

Data Availability Statement: Not applicable.

Acknowledgments: The authors would like to thank the reviewers for their important suggestions and careful reading of our manuscript. The authors would like to thank M. Kian, who let us know the essential estimation for the symmetric function in Lemma 6.

Conflicts of Interest: The authors declare no conflict of interest.

\section{References}

1. Rényi, A. On measures of entropy and information. In Proceedings of the Fourth Berkeley Symposium on Mathematical Statistics and Probability, Volume 1: Contributions to the Theory of Statistics; University of California Press: Berkeley, CA, USA, 1961; Volume 1, p. 547.

2. Shannon, C.E. A mathematical theory of communication. Bell Syst. Tech. J. 1948, 27, 379-656. [CrossRef]

3. Tsallis, C. Possible generalization of Bolzmann-Gibbs statistics. J. Stat. Phys. 1988, 52, 479-487. [CrossRef]

4. Beck, C.; Schlögl, F. Thermodynamics of Chaotic Systems: An Introduction; Cambridge University Press: Cambridge, UK, 1993.

5. Conroy, J.M.; Miller, H.G.; Plastino, A.R. Thermodynamic consistency of the q-deformed Fermi-Dirac distribution in nonextensive thermostatics Phys. Lett. A 2010, 374, 4581-4584. [CrossRef]

6. Furuichi, S.; Mitroi, F.-C. Mathematical inequalities for some divergences. Phys. A 2012, 391, 388-400. [CrossRef]

7. Furuichi, S.; Minculete, N. Refined Young inequality and its application to divergences. Entropy 2021, 23, 514. [CrossRef] [PubMed]

8. Sababheh, M.; Choi, D. A complete refinement of Young's inequality. J. Math. Anal. Appl. 2016, 440, 379-393. [CrossRef]

9. Butt, S.I.; Mehmood, N.; Pečarić, D.P.; Pečarić, J.P. New bounds for Shannon, relative and Mandelbrot entropies via AbelGontscharoff interpolating polynomial. Math. Inequal. Appl. 2019, 22, 1283-1301. [CrossRef]

10. Tohyama, H.; Kamei, E.; Watanabe, M. The $n$-th residual relative operator entropy $\mathfrak{R}_{x, y}^{[n]}(A \mid B)$. Adv. Oper. Theory 2021, 6, 18 . [CrossRef]

11. Isa, H.; Kamei, E.; Tohyama, H.; Watanabe, M. The $n$-th relative operator entropies and the $n$-th operator divergences. Ann. Funct. Anal. 2020, 11, 298-313. [CrossRef]

12. Kittaneh, F.; Manasrah, Y. Improved Young and Heinz inequalities for matrix. J. Math. Anal. Appl. 2010, 361, 262-269. [CrossRef]

13. Alzer, H.; da Fonseca, C.; Cec, A.K. Young-type inequalities and their matrix analogues. Linear Multilinear Algebra 2014, 63, 622-635. [CrossRef]

14. Cartwright, D.I.; Field, M.J. A refinement of the arithmetic mean-geometric mean inequality. Proc. Amer. Math. Soc. 1978, 71, 36-38. [CrossRef]

15. Furuichi, S.; Ghaemi, M.B.; Gharakhanlu, N. Generalized reverse Young and Heinz inequalities. Bull. Malays. Math. Sci. Soc. 2019, 42, 267-284. [CrossRef]

16. Sababheh, M.; Moslehian, M.S. Advanced refinements of Young and Heinz inequalities. Number Theory 2017, 172, 178-199. [CrossRef]

17. Alakhrass, M.; Sababheh, M. Matrix mixed mean inequalities. Results Math. 2019, 74, 2. [CrossRef]

18. Cover, T.M.; Thomas, J.A. Elements of Information Theory, 2nd ed.; Wiley-Interscience: Hoboken, NJ, USA, 2006.

19. Sababheh, M. Log and harmonically log-convex functions related to matrix norms. Oper. Matrices 2016, 10, 453-465. [CrossRef] 